\title{
1 Zur Soziologie des Nichtwissens
}

Der Begriff des Nichtwissens hat sich in den letzten Jahren zu einem Gegenstand soziologischer Diskurse entwickelt. Als inhärenter Bestandteil jeglicher Wissensproduktion hat der Begriff insbesondere im Kontext von Ökologie-, Technik- und Risikodiskursen an Bedeutung gewonnen (Beck $\&$ Wehling 2012; Marchant, et al. 2007). Dabei verweist er auf unbekannte und unerwartete Handlungs- und Entscheidungsfolgen jenseits kalkulierbarer Risiken und abschätzbarer Ungewissheiten. Er gilt als mit wissenschaftlicher Wissensproduktion und technologischer Entwicklung untrennbar verknüpft (Böschen \& Wehling 2012), als Schatten jeglichen Wissens (Japp 2002) und als inhärent in jeglicher Entscheidungsfindung, sozialer Interaktion und Alltagskommunikation vorhanden (Gross \& McGoey 2015). Aus diesen Auffassungen von Nichtwissen hat sich eine ,Soziologie des wissenschaftlichen Nichtwissens' oder im Englischen die ,sociology of ignorance' entwickelt. Diese beschäftigt sich damit, wie Nichtwissen unbeabsichtigt entsteht, wie Wissen bewusst ignoriert wird, und wie die Wissenschaft und - was insbesondere Gegenstand der vorliegenden Studie ist — die Gesellschaft mit Nichtwissen umgeht (Böschen \& Wehling 2012, 321; Gross \& McGoey 2015).

Ausgangslage dieses Bandes ist die These, dass nichtintendiertes Nichtwissen in aktuellen technowissenschaftlichen Diskursen eine neue und zentrale Aufmerksamkeit erhält und damit verknüpft, eine wissenschaftsund politikübergreifende Notwendigkeit zum Eingreifen abgeleitet wird. Dies steht im Gegensatz zu früheren Diskursen, in welchen Nichtwissen meist ignoriert oder als ,Restrisiko' ausgeblendet worden ist (Wehling 2006). Am Beispiel des Nanotechnologiediskurses in Deutschland, Großbritannien, den Vereinigten Staaten und auf der Ebene der Europäischen Union wird hier gezeigt, dass der neue Fokus auf Nichtwissen und die daraus abgeleitete Notwendigkeit zum Eingreifen - hier mit dem Begriff des Lenkens umschrieben und diskursanalytisch in Anlehnung an Foucault (Foucault 1978) als Dispositiv bezeichnet wird, also als ein strategischer MachtWissens-Komplex, der immer Neues möglich macht — darauf abzielt, Nichtwissen definierbar, kalkulierbar und regulierbar zu machen (siehe Kapitel 1.2). Dies geschieht mittels dreier zentraler Strategien: 1) dem Abschätzen, 2) dem Regulieren und 3) der Partizipation. 
Die drei Strategien werden in dieser Arbeit analytisch als Diskurse untersucht und inhaltlich breit verstanden. Somit umfasst der Abschätzungsbegriff nicht nur die schriftliche Analyse potenzieller nachteiliger Implikationen und Risiken der traditionellen Technikfolgenabschätzung (TA) oder der Begleitforschung, sondern ebenso Nutzenanalysen, das Einsetzen von Gremien und die Etablierung akademischer Zentren und spezifischer Projekte. Das im Nanotechnologiediskurs feststellbare umfangreiche Bestreben nach Abschätzung wird als ein ,Dispositiv' bezeichnet, das zu einem umfassenden Abschätzungsboom beigetragen hat. Dabei lässt sich festellen, dass nicht nur traditionellerweise in die Abschätzung neuer Technologien involvierte Akteure, wie die Technikfolgenabschätzung und die Begleitforschung in Abschätzungsaktivitäten involviert sind, sondern die verschiedensten gesellschaftlichen Akteure. Diese gesellschaftliche Distribution von Abschätzungsaktivitäten wird hier auf den im Kontext von Nichtwissen beschriebenen Kompetenz- oder Autoritätsverlust von gesellschaftlichen Institutionen zurückgeführt. So verlieren in Nichtwissensdiskursen die einzelnen gesellschaftlichen Institutionen also ihre traditionellerweise vorhandenen eindeutig zuordenbaren Verantwortlichkeiten (Böschen \& Wehling 2004).

Auch der Regulierungsbegriff wird hier breit verstanden. Er umfasst hier sowohl die gesetzliche Regulierung als auch weniger bindende Formen der freiwilligen Regulierung. Ebenso unter den Regulierungsbegriff subsumiert werden selbstregulatorische Lenkungsansätze, die von regierungsexternen Akteuren beispielsweise als Risikomanagementsysteme, Handlungsanweisungen, Zertifizierungssysteme und Verhaltenskodizes im Zuge eines ,selbstregulatorischen Nichtwissensmanagements' (Lösch 2012) etabliert werden. In diesem Verständnis beschränkt sich das Regulieren nicht alleine auf gesetzliche Interventionen des sogenannt ,harten Rechtes' (hard law), sondern umfasst auch freiwillige ,eingrenzende und risikominimierende' ebenso wie ,fördernde und ermöglichende' Maßnahmen (Bora 2012, 352) des sogenannt, weichen Rechts' (soft law) und der gesellschaftlichen Selbstregulierung.

Unter dem Begriff der Partizipation wird hier schließlich die ,Externalisierung von Nichtwissen' (Lösch 2012) in unterschiedliche Foren und Formen der Beteiligung verstanden. Dies betrifft einerseits das Einbinden von Akteuren und Exponenten unterschiedlicher involvierter Organisationen in dialogische und deliberative Verfahren der Anspruchsgruppenpartizipation und andererseits dasjenige der Bevölkerung in solche der zivilgesellschaftlichen Partizipation. Solche Verfahren verfolgen Ziele wie Ver- 
netzung, Meinungsbildung und Beratung ebenso wie die Einbindung von Anspruchsgruppen und der Zivilgesellschaft in die Entscheidungsfindung in wissenschafts- und technikpolitische Fragestellungen.

Bei diesen drei Strategien handelt es sich um eine analytische Trennung von Phänomenen, die sich in der Realität nicht trennscharf voneinander abgrenzen lassen. Beispielsweise kann die Gremienarbeit sowohl Abschätzungs- als auch partizipative oder regulatorische Komponenten umfassen. Ansätze wie Risikomanagementverfahren beinhalten sowohl Facetten der Abschätzung als auch solche der Selbstregulierung. In einem bestimmten Maß bedingen sowohl das Regulieren wie auch die Partizipation die Abschätzung. Ohne vorgängige Analysen des Gegenstands kann weder reguliert noch können Anspruchsgruppen oder die Zivilgesellschaft in partizipative Verfahren eingebunden werden. Ebenso sind die Ergebnisse partizipativer Verfahren häufig wiederum Studien, die auch der Abschätzung zugeordnet werden können. Dennoch wird hier die Haltung vertreten, dass eine analytische Trennung in einzelne Lenkungsstrategien für das Verständnis und die Darstellung des Lenkungsdispositivs im Nanotechnologiesiskurs unabdingbar sind.

Die nachfolgende soziologische Analyse fokussiert also die drei Lenkungsstrategien; 1) Abschätzen, 2) Regulieren und 3) Partizipation. Die Strategien werden analytisch als einzelne Diskurse behandelt und als solche untersucht. Übergreifend werden sie jedoch als Strategien des Nanotechnologiediskurses diskutiert. Im Zentrum der nachfolgenden Diskursanalyse steht die Frage, wie zeitgenössische Wissensgesellschaften mit den hier unter dem Begriff des ,Nichtwissens' bezeichneten Konstellationen und Situationen des Versagens etablierter wissenschaftlicher Ansätze in der Einschätzung potenzieller Nutzen und nachteiliger Implikationen ${ }^{1}$ aktueller technowissenschaftlicher Entwicklungen umgehen. Der Umgang mit Nichtwissen, das mit Implikationen technowissenschaftlicher Forschung und Entwicklung verknüpft ist, wird hier also als diskursives, kultur- und politikrelevantes Phänomen analysiert.

Eine solche Perspektive stellt die Begriffe Politik, Wissenschaft und Technik in einen neuen, vielleicht ungewohnten Zusammenhang. So geht

1 Diese Studie verwendet bewußt die Bezeichnung 'nachteilige Implikationen' anstelle von 'Risiko'. Während der Risikobegriff für ein kalkulierbares Schadenszenario verwendet wird, steht die Bezeichnung 'nachteilige Implikationen' für ein breiteres Spektrum kalkulierbarer und nichtkalkulierbarer Schadensapekte (Böschen \& Wehling 2004). 
es hier nicht um die politische Beeinflussung der Wissenschaft, im Sinne des unter dem Begriff der ,neuen Wissenschaftskultur' (Maasen \& Weingart 2007) subsumierten wissenschafts- und forschungspolitischen Phänomens einer verstärkten Ökonomisierung, Managerialisierung und Politisierung von Universitäten und akademischer Forschung (vgl. Felt \& Fochler 2010b; Gibbons, et al. 1994; Irwin 2006; Jasanoff 2005; Weingart 2001). Gegenstand dieser Studie ist auch nicht die Forschungsförderung oder eine Analyse des Verhältnis zwischen Wissenschaft und Politik (z.B. Felt, et al. 2007; Luhmann 1984; Stehr 2002; Stichweh 1988; Weingart 2003) oder das Problem der wissenschaftlichen Politikberatung bzw. dasjenige der Expertise, das unter den Aspekten Wissen, Macht, Einflussnahme und Demokratisierung von Wissenschaft und Technik breit diskutiert wird (z.B. Bogner 2007; Jasanoff 1990; Maasen \& Weingart 2005; Weingart 2006). Im Fokus der vorliegenden Untersuchung steht vielmehr die von Böschen und Wehling (2012) aufgeworfene Frage nach dem gesellschaftlichen Umgang mit aktueller technowissenschaftlicher Forschung und Entwicklung und dem damit verbundenen Nichtwissen.

\subsection{Die wissenssoziologische Analyse von Nichtwissensdiskursen}

Diese Untersuchung basiert auf dem methodischen Ansatz der wissenssoziologischen Diskursanalyse (Keller 2011). Dieser Ansatz fasst Diskurse als ,mehr oder weniger öffentliche, geplante und organisierte Diskussionsprozesse' auf, die sich auf bestimmte, gesellschaftlich relevante Themen beziehen (ibid.). Er eignet sich insbesondere zur Untersuchung von Fragen des sozialen Wandels, die unter Begriffen wie demjenigen der Wissensgesellschaft, der Risikogesellschaft oder wie in der vorliegenden Untersuchung unter dem Wandel der sozialen Aufmerksamkeit von Wissen hin zu Nichtwissen (vgl. dazu Kapitel 1.2) diskutiert werden (Keller 2011).

Bei diesem Ansatz werden Diskurse als konstituierende Elemente der ,soziale[n] Realität von Phänomenen' und als eine soziale Praxis angesehen, die sich aus verstreuten diskursiven Ereignissen zusammensetzt (Keller 2011, 7, 236). ${ }^{2}$ Die wissenssoziologische Diskursanalyse orientiert sich

2 Diese diskursiven Ereignisse umfassen mündliche und schriftliche kommunikative Äußerungen, wie z.B. Vorträge und Berichte und können zeitlich, räumlich und sozial auch weit auseinander liegen. Nach Keller (2011) sind diskursive Ereignisse von unterschiedlichsten sozialen Akteuren für diverse Publika hergestellt, enthalten 
dabei am Ansatz Foucaults (1972) und berücksichtigt sozialkonstruktivistische Ansätze, wie Berger und Luckmann (1969), kulturanalytische Ansätze, wie die Laborstudien der sozialwissenschaftlichen Wissenschaftsund Technikforschung ${ }^{3}$ und material-semiotische Ansätze der AkteurNetzwerk Theorie (Latour 1987; Latour 2005; Law \& Hassard 1999). Sie geht dabei von der sozialen Konstruktion von Wirklichkeit durch Akteure und der Konstruiertheit jeglichen Wissens aus. Insbesondere ermöglicht diese Methode auch die Analyse von performativen Ansätzen der Wissensproduktion und der Bedeutung, die sich aus den Interaktionen mit Artefakten und Materialitäten ergeben. Der Akteursbegriff umfasst hier neben Einzelakteuren immer auch Kollektive, wie Akteursgruppen, Organisationen und Institutionen. Dabei fokussiert die wissenssoziologische Diskursanalyse die gesellschaftliche Wirkung nicht nur durch die soziale Konstruktion der Akteure, sondern auch durch deren Praktiken und Inhalte (Keller 2011, 95). Zudem beschränkt sich die Wissenssoziologische Diskursanalyse nicht nur auf aktuelle Diskurse und deren gegenwärtige Prägung, sondern rekonstruiert auch vergangene Diskurse, den zeitlichen Verlauf und die Ursprünge bestimmter Diskurse mit einem soziohistorischen Ansatz. Dabei stehen Fragen nach kontingenten Entwicklungen, Interessen, Strategien und Handlungsressourcen der beteiligten Akteure, Organisationen und Institutionen im Zentrum der Analyse und es werden allgemeine theoretische Kategorien und Thesen über typisierbare Formen und Mechanismen einzelner Diskurse entwickelt (Keller 2011, 17). Damit stehen bei Keller (2011) vergleichbar mit den in Kapitel 1.3 näher beschriebenen kulturanalytischen Ansätzen der sozialwissenschaftlichen Wissenschafts- und Technikforschung — wie Knorr Cetina's (2002) Wissenskulturen und Jasanoff's (2005) politische Kulturen - Akteure, Praktiken und Inhalte im Fokus der Diskursanalyse. Bezüglich des methodischen Vorgehens schlägt Keller (2011), eine mehrere Ansätze der qualitativen Sozial-

jedoch dennoch einen typisierbaren Kerngehalt, eine typische „Aussage" im Sinne Foucaults und sind demnach Teil ein und desselben Diskurses.

3 Die zwischen Ende der 1970er Jahren und den frühen 1980er Jahren von verschiedenen Forschenden durchgeführten sozialwissenschaftlichen ,Laborstudien' (z.B. Knorr Cetina 1981; Knorr Cetina 1995; Latour 1987; Latour \& Woolgar 1979; Lynch 1985) gelten als grundlegend für die konstruktivistische Auffassung der akademischen Wissensproduktion. Ihr Ansatz liegt darin, naturwissenschaftliche Erkenntnis als praktische, soziale Tätigkeit direkt am Ort ihres Entstehens; dem Labor, zu beobachten (vgl. Wehling 2006). 
forschung umfassende Herangehensweise vor, die unterschiedliche Zugänge der Datenerhebung ermöglicht.

Vor diesem Hintergrund analysiert diese Arbeit Nichtwissensdiskurse im Bereich der Nanotechnologie entlang der drei analytischen Kategorien 1) Akteure, 2) Praktiken und 3) Inhalte (vgl. auch Kapitel 1.3). Für diese Analyse ist ein Methodenmix basierend auf leitfadengestützten Experteninterviews mit Schlüsselakteuren, Dokumentenanalyse und kurzzeitigen, teilnehmenden Beobachtungen an Sitzungen und an Präsentationen von zentralen Gremien und Kommissionen durchgeführt worden. So sind mit zentralen in den Nanotechnologiediskurs involvierten Akteuren aus Deutschland, Großbritannien, der Europäischen Union und den Vereinigten Staaten 74 qualitative, leitfadengestützte Experteninterviews nach Bogner and Menz (2009) geführt worden (siehe Liste in Kapitel 8). Diese sind aufgenommen, transkribiert und inhaltsanalytisch ausgewertet worden (Lamneck 1995; Mayring 1988). Mittels der hauptsächlich in den Jahren 2008 und 2009 geführten Experteninterviews und Beobachtungen ist eine Momentaufnahme des Nanotechnologiediskurses aus diesem Zeitraum entstanden, der mit der Analyse älterer und aktuellerer Dokumente in einen Kontext gestellt worden ist. Ausgewertete Dokumente umfassen alle Arten von Abschätzungsberichten (siehe Kapitel 2), regulatorischen Dokumenten (siehe Kapitel 3), Protokollen und Abschlussberichten von partizipativen Veranstaltungen und Gremien (siehe Kapitel 4). Auch diese sind inhaltsanalytisch nach Lamneck (1995) und Mayring (1988) ausgewertet worden. Die teilnehmenden Beobachtungen nach Emerson, et al. (2001) sind kurzzeitig an für die Analyse wesentlichen Sitzungen, Konferenzen und Präsentationen erfolgt und sind in Feldnotizen (Emerson, et al. 2011) festgehalten worden, die wiederum nach der Methode von Mayring (1988) inhaltsanalytisch ausgewertet worden sind. Ausführlichere methodische Bemerkungen folgen in der Einführung zu den jeweiligen Kapitel 2, 3 und 4. In den empirischen Kapiteln (2-4) werden neben der Darlegung und der Interpretation der in der Diskursanalyse gewonnenen Erkenntnisse auch sprachlich bereinigte Interviewsequenzen als Zitate direkt in den Text eingefügt und interpretiert. Dies dient der Erläuterung und der Untermauerung der im Fließtext vertretenen Argumente. Auch ist der Forschungsprozess, während des Vorgehens kontinuierlich reflektiert und angepasst worden (Keller 2011, 268). Texte, Praktiken und Artefakte werden dabei nicht als ,Produkte subjektiver oder objektiver Fallstrukturen', sondern als Ausdruck, gesellschaftlicher Wissensordnungen und -politiken' betrachtet (Keller 2011, 275). 
Der dieser Diskursanalyse zugrunde liegende empirische Fall ist die Nanotechnologie. Unter diesem Begriff ist in westlichen und östlichen Industrienationen die Forschung und Entwicklung auf kleinstmolekularer Ebene als eine Spitzentechnologie eingeführt worden. Diese verheißt für unterschiedlichste gesellschaftliche Bereiche nützliche Anwendungen und wird von einer äußerst visionären Rhetorik begleitet (siehe Kapitel 1.4). Der Diskurs um potenzielle nachteilige Implikationen hat im Vergleich zu vorangegangenen Technikdiskursen zeitlich relativ früh in der technologischen Entwicklung eingesetzt. So sind erste Technikfolgenabschätzungsstudien und Implikationsanalysen nahezu zeitgleich mit der Publikation nationaler Initiativen, oder im Fall von Großbritannien sogar davor erfolgt (siehe Kapitel 2.1).

Die Nanotechnologie steht hier als Fall eines technowissenschaftlichen Diskurses, an dem der gesellschaftliche Umgang mit Nichtwissen diskutiert wird. Dabei werden im Rahmen eines internationalen Vergleichs die die Nanotechnologie intensiv fördernden Industrienationen, Vereinigte Staaten, Großbritannien und Deutschland, und die supranationale Ebene der Europäischen Union analysiert. Damit werden zugegebenermaßen unterschiedliche politische Gebilde untersucht, die einem traditionellen, auf maximale Homogenität der Stichproben ausgerichteten Vergleichsansatz nicht standhalten. Mit der methodologischen Orientierung an den Kulturvanalysen der sozialwissenschaftlichen Wissenschafts- und Technikforschung (z.B. Jasanoff 2005), die nicht Stichproben, sondern vielmehr Fälle bzw. Diskurse analysieren, lassen sich auch äußerst heterogene Phänomene vergleichen, wie in diesem Fall Nationalstaaten mit supranationalen Organisationen. ${ }^{4}$ Diese Vergleichsanlage mit der Europäischen Union, den beiden (Noch-) Mitgliedsstaaten Deutschland und Großbritannien sowie dem Nichtmitgliedsstaat der Vereinigten Staaten zielt darauf ab, die in drei in der nanowissenschaftlichen Forschung und Entwicklung führenden Nationalstaaten ergriffenen Lenkungsmaßnahmen in ihren individuellen kulturellen Kontexten zu untersuchen. Die Analyse der Unionsebene wird hier für das Verständnis der in den beiden Mitgliedsstaaten ergriffenen Maßnahmen als unabdingbar angesehen. Im Zentrum dieser Untersuchung stehen dabei die Strategien, die diese Länder und die Union im Umgang

4 Damit wird hier Jasanoff's (2005) Feststellung gefolgt, dass sich mit diesem methodologischen Ansatz alles vergleichen lässt. 
mit dem mit der Nanotechnologie verbundenen Nichtwissen zeigen: 1) dem Abschätzen, 2) dem Regulieren und 3) der Partizipation.

Die Untersuchung hat gezeigt, dass Forschung und Entwicklung in den nationalen Forschungsinstitutionen beginnen. Diese auf nationaler Ebene stattfindenden Vorarbeiten sind für unterschiedlichste Lenkungsmaßnahmen unabdingbar, mit welcher neben der nationalen auch die Unionsebene ins Spiel kommt. Es hat sich also gezeigt, dass die Diskurse auf nationaler Ebene beginnen und die Unionsebene sich mittels spezifischer Maßnahmen wie Forschungsförderung und Regulierung in die Diskurse einbringt. Um diese spezifischen Maßnahmen sowohl auf nationaler als auch auf supranationaler Ebene zu verstehen, erfolgt in den einzelnen Länderkapiteln immer auch ein kurzer historischer Abriss über die jeweils spezifische Entwicklung des Nanotechnologiediskurses. Diese Studie zeigt weiter, dass im Nanotechnologiediskurs die individuellen nationalstaatlichen Kontexte verschwimmen und die supra- und internationale Kooperation an Bedeutung gewinnen (vgl. Kapitel 5).

Die Frage nach der Behandlung von Nichtwissen eröffnet ein breites und komplexes Untersuchungsfeld, für dessen Bearbeitung verschiedene soziologische Zugänge und Traditionen relevant werden. Diese führen von der Kultursoziologie über die sozialwissenschaftliche Wissenschafts- und Technikforschung (STS) und die Wissenschafts- und Techniksoziologie bis hin zur politischen Soziologie.

Das nachfolgende Kapitel 1.2 erläutert die dieser Studie zugrunde liegenden zentralen Begrifflichkeiten des Nichtwissens und des Lenkens. Das Kapitel 1.3 gibt einen Überblick über die dieser Arbeit zugrunde liegenden Theorien und Konzepte der vergleichenden Kulturanalysen der Wissenschafts- und Technikforschung, wie Wissenskulturen (Knorr Cetina 2002) und politische Kultur (Jasanoff 2005). Im Kapitel 1.4 folgen einige Bemerkungen zum Fall der Nanotechnologie als technowissenschaftliches Phänomen. Kapitel 1.5 erläutert die dieser Studie zugrunde liegenden Thesen, die Fragestellung und das Kapitel 1.6 schließlich erläutert die Gliederung des Bandes.

\subsection{Nichtwissen lenken}

Mit ,Nichtwissen' und ,Lenken' liegt der Fokus dieses Bandes auf zwei grundsätzlich unterschiedlichen Begriffen. Während ,Lenken' für eine koordinierende, steuernde und zielgerichtete Handlung steht, bezeichnet 
Nichtwissen das Unbekannte, Unklare und Ungewisse. Angesichts dieser Divergenz stellt sich die Frage, wie Nichtwissen gelenkt werden kann, bzw. wie Nichtwissen zum Gegenstand einer Lenkungskultur werden kann. Das Zusammenwirken der beiden Begriffe ergibt sich aus der These dieses Bandes, die besagt, dass in technowissenschaftlichen Diskursen angesichts des verstärkten Fokus auf Nichtwissen ein Lenkungsdispositiv entsteht. In der wissenssoziologischen Diskursanalyse (siehe Kapitel 1.1), charakterisiert Keller $(2011,258)$ ein Dispositiv als einen spezifischen Unterbau der Herstellung des Diskurses und der Problembearbeitung, welche die diskursführenden sozialen Akteure schaffen. Dispositive stellen also die Mittel dar, mittels welchen die Diskurse ihre Macht entfalten können und sie dienen dazu, als zuständige Stellen zwischen den diskursiven Äußerungen und Praktiken zu vermitteln. Dabei stellt ein Dispositiv den ,institutionellen Unterbau' eines Diskurses, also die Gesamtheit der ,personellen, materiellen, kognitiven, handlungspraktischen und normativen Infrastruktur' dar (ibid.). Ebenso bestimmt das Dispositiv die Umsetzung seiner angebotenen ,Problemlösung ${ }^{6}$ in einem spezifischen Praxisfeld (Keller 2011).

Diese Studie basiert auf der Annahme, dass in Nichtwissensdiskursen ein Lenkungsdispositiv entsteht, das darauf abzielt, Nichtwissen mittels Abschätzungs-, Regulierungs- und Partizipationsaktivitäten definierbar, kalkulierbar und regulierbar zu machen. Das Auftreten eines Bewusstseins über Wissenslücken, so unspezifisch sie auch sein mögen, führt also zu einer sozialen Handlungskoordination im Sinne eines Steuerungs- oder Lenkungshandelns. Diese durch das Nichtwissen induzierte Handlungskoordination erfolgt dabei im Kontext inkrementeller, vieldeutiger aber auch zufällig und situativ auftretender Muster und Verbindungen, die hier als ,Lenkungskultur' definiert werden (zum Begriff der Lenkungskultur siehe Kapitel 1.3).

Nichtwissen (übersetzt als ,ignorance', ,unknown' oder ,nonknowledge') hat sich in den letzten Jahren als Gegenstand der Soziologie etabliert (Böschen \& Wehling 2012, 317). Insbesondere in der soziologischen Risikoforschung lässt sich der Trend eines verstärkten Fokus auf Nichtwissen feststellen. Diese soziologische Strömung baut einerseits auf Theorien der ökologischen Kommunikation (Luhmann 1986), des Risikos 
(Luhmann 1991), ${ }^{5}$ bzw. der reflexiven Modernisierung (Beck 1993; Beck, et al. 1996) auf. Ebenso basiert sie auf Technik- und Risikotheorien der Wissenschafts- und Technikforschung (z.B. Giddens 1992; Ravetz 1987; Smithson 1985; Smithson 1989; Wynne 1991a) und auf den Laborstudien der sozialwissenschaftlichen Wissenschafts- und Technikforschung (z.B. Knorr Cetina 2002). ${ }^{6}$

Aus diesen Auffassungen von Nichtwissen hat sich eine ,Soziologie des wissenschaftlichen Nichtwissens' oder im angelsächsischen Raum eine ,sociology of ignorance' entwickelt (vgl. S. 8). Darin wird Nichtwissen entlang der drei Dimensionen 1) Intentionalität, 2) Spezifizierung und 3) Temporalität verstanden. Die erste Dimension unterscheidet zwischen unbeabsichtigtem und gewolltem Nichtwissen. Sie steht also für die Abgrenzung zwischen nicht intendierten Wissenslücken, wie solchen nach den Umweltauswirkungen bestimmter Nanomaterialien und bewusstem, strategischem oder gezieltem Ignorieren von verfügbarem Wissen. Dies umfasst auch das Recht auf Nichtwissen, wie beispielsweise dasjenige bezüglich prädiktiver genetischer Diagnosen in der Humanmedizin (z.B. McGoey 2012; McGoey 2014). ${ }^{7}$ Die zweite Dimension steht für die Unterscheidung zwischen gewusstem und unerkanntem Nichtwissen, also zwischen bekannten Wissenslücken und unbekannten Ungewissheiten. Die dritte Dimension schließlich steht für die zeitliche Differenzierung von Nichtwissen, zwischen einem temporär unterstellten ,Noch-Nicht-Wissen' und einem als grundsätzlich unauflösbar eingeschätzten ,Nichtwissen-Können'.

5 Insbesondere die breite sozial- und kulturwissenschaftliche Kritik an der ,technischen Verkürzung' und der als reduktionistisch wahrgenommenen Logik des Risikobegriffs, hat zu einem Bedeutungszuwachs alternativer Begrifflichkeiten und Konzepte geführt (vgl. Beck 1986; Collingridge 1980; Douglas \& Wildavsky 1982; Ravetz 1987; Wynne 1987).

6 Mit der Anlehnung der Nichtwissenssoziologie an die Laborstudien, die das Ziel verfolgen, naturwissenschaftliche Erkenntnis als praktische, soziale Tätigkeit direkt am Ort ihres Entstehens; dem Labor, zu beobachten (vgl. Wehling 2006), wird Nichtwissen auch hinsichtlich seines kontingenten, konstruktiven und selektiven Charakters verständlich und analysierbar (vgl. Wehling 2001).

7 Im Englischen werden diese beiden Formen von Nichtwissen semantisch unterschieden. So stehen die Begriffe ,non-knowledge' oder , unknown' für nichtintendiertes Nichtwissen und der Begriff , ignorance' für intendiertes Nichtwissen (Gross \& McGoey 2015; Wehling 2006). 
Wehling (2001, 2003, 2004) und Böschen und Wehling (2004, 2012) stellen die Soziologie des Nichtwissens in die Nähe der Wissenssoziologie und sprechen diesbezüglich auch von einer empirisch orientierten Wissenssoziologie des Nichtwissens. ${ }^{8}$ Diese untersucht Fragen nach dem Entstehen, den Bedingungen, den Funktionen und der gesellschaftlichen Relevanz von Nichtwissen. Zudem fragt die Soziologie des Nichtwissens nach dem Umgang von Gesellschaften, Institutionen und sozialen Akteuren mit Nichtwissen und den Konsequenzen, die diese daraus ziehen (Wehling 2006). Ein weiterer Bereich dieser Forschung beschäftigt sich damit, wie Wissen gezielt oder strategisch ignoriert wird und wie dies die gesellschaftlichen Machtstrukturen und die politische Steuerung von Wissen beeinflusst (McGoey 2012).

In der dieser Studie zugrunde liegenden Leseart wird Nichtwissen als Ergebnis wissenschaftlicher Wissensproduktion und technologischer Entwicklungen und als grundsätzlich mit Wissen in vielschichtiger Weise untrennbar verknüpft aufgefasst. ${ }^{9}$ Unter dieser Auffassung erscheint Nichtwissen weniger als ,bekannte' oder ,spezifische' Wissenslücke im Sinne von Merton (1987) oder Popper (1989), die in der breit hinterfragten Idee des kumulativen und endlosen Wissensfortschritts begründet liegt. Ebenso wenig wird Nichtwissen hier im Sinne eines strategischen, bewussten oder intendierten Ignorierens von bestehendem Wissen verstanden (Gross \& McGoey 2015). Vielmehr wird Nichtwissen im Nanotechnologiediskurs hier in Anlehnung an Wehling (2006) als eine unspezifische und unerkannte, bzw. als eine nichtintendierte Wissenslücke aufgefasst. Ebenso wenig wird Nichtwissen in dieser Lesart systemtheoretisch als kontextunabhängige Schattenseite des Wissens verstanden (Luhman 1992), da auch dieses Verständnis von Nichtwissen implizit auf eine spezifische Wissenslücke hindeutet (vgl. Böschen \& Wehling 2012). Auch nicht unter den Be-

8 Zur Wissenssoziologie siehe Maasen (1999).

9 Damit ist die Produktion von Wissen grundlegend mit der Erzeugung von Nichtwissen verbunden, worauf bereits Ludwik (Fleck 1935) hingewiesen hat (vgl. auch Böschen \& Wehling 2012, 321). Nichtwissen stellt also nicht ein temporäres und lösbares Begleitphänomen des wissenschaftlich-technischen Fortschritts dar, sondern wird als inhärent mit diesem verknüpft ist, bzw. durch diesen laufend erhöht aufgefasst. Das in Wissensgesellschaften der reflexiven Moderne zunehmende Bewusstsein über die Verknüpfung von Nichtwissen mit dem wissenschaftlichen Wissensgewinn trägt zur Entwicklung neuartiger wissenschaftlicher, politischer und gesellschaftlicher Transformationen, Verschiebungen und Brüche bei (vgl. Wehling 2003). 
griff des Nichtwissens subsumiert wird hier das im Kontext des Public Understanding of Science (siehe Kapitel 4) festgestellte mangelnde Verständnis der Bevölkerung in wissenschafts- und technikbezogenen Inhalten (Wehling 2004). Dieses mangelnde Verständnis der Bevölkerung wird unter dem Begriff der ,public ignorance of science' beschrieben (vgl. Michael 1986).

Im Gegensatz zu Risikokonstellationen, in welchen ein primär wissenschafts- und technikbasierter Ansatz des Umgangs mit Entscheidungsfolgen besteht, beschreiben Böschen and Wehling (2012) im Umgang mit Nichtwissenssituationen eine Verlagerung der Verantwortung von der Wissenschaft auf die politische und weiter auf die gesamtgesellschaftliche Ebene. Dies führt zu einer Öffnung des wissenschaftlichen Expertendiskurses für weitere Anspruchsgruppen, für neue Formen der Wissensproduktion und des politischen Handelns, ebenso wie zur Etablierung neuartiger institutioneller Konstellationen (Wehling 2004). Damit bleibt die Beurteilung aktueller Spitzenforschungsfelder und von sich an naturwissenschaftlichen Konvergenzzonen interdisziplinär herausbildenden großtechnischen Entwicklungen hoher Komplexität nicht mehr länger Aufgabe der Wissenschaft allein. Vielmehr verteilt sich diese auf weitere gesellschaftliche Bereiche wie die Wirtschaft, die Politik und die Gesamtgesellschaft (z.B. Stehr 2002; Weingart 2001).

Im Unterschied zu Wissensdiskursen verschiebt sich in Nichtwissensdiskursen der wissenschafts- und gesellschaftspolitische Fokus von epistemischen Aspekten hin zu fundamentalen Strukturen, Werten und Haltungen einer Gesellschaft an sich. Dieser Fokus umfasst dabei Fragen wie diejenige nach einer politischen Steuerung von Forschung (Weingart 2001), nach demokratischer Kontrolle von Wissenschaft (z.B. Nowotny, et al. 2001) und solche nach der Einbindung der Zivilgesellschaft in die wissenschafts- und technikbezogene Entscheidungsfindung (z.B. Jasanoff 2005). Dabei findet also eine ,Politisierung' von Nichtwissen statt, die Wehling $(2003,36)$ in Anlehnung an Smithson (1993), Stocking/Holstein (1993) und Weingart (2003) als ,politicization of ignorance' bezeichnet. Insbesondere in technowissenschaftlichen Bereichen führt die Politisierung des Nichtwissens dazu, dass die Wissenschaft ihre Legitimität und ihre für Wissensaspekte traditionell vorhandene Deutungshoheit verliert. Damit gewinnen in Nichtwissensdiskursen politische und gesellschaftliche Foren und Formen der Bewertung und Abschätzung zunehmend an Bedeutung (Wehling 2003). 
Ziel dieser Studie ist es, die bisher wenig beachtete soziologische Perspektive und Konzeptualisierung von Nichtwissen für die Wissenschaftsund Technikforschung fruchtbar zu machen. Damit soll ein Beitrag zu einer empirisch orientierten Wissenssoziologie des Nichtwissens geleistet werden, welche die Funktionen und Relevanzen von Nichtwissen in gesellschaftlichen Kontexten zum Gegenstand nimmt.

Neben dem Nichtwissen beschreibt der zweite zentrale Begriff dieser Arbeit das Lenken. Dieser Begriff wird hier für die im Englischen unter dem vieldeutigen Begriff der ,Governance' (Offe 2008) subsumierten mannigfaltigen Formen und Mechanismen der gesellschaftlichen Steuerung und der Koordination kollektiven Handelns und Eingreifens verstanden. Aufgrund seiner vieldeutigen Verwendung für Institutionen und Prozesse wird ,Governance' auch als vages Konzept, als ,analytische Perspektive' (Lösch 2014) oder als ,empty signifier' beschrieben (Offe 2008). Um dieser und der sprachlichen Vieldeutigkeit des Governance-Begriffs auszuweichen, wie dem Fehlen von Gegenbegriffen, von Synonymen und seiner ungeklärten Übersetzung ins Deutsche, wird hier dem Lenkungsbegriff der Vorzug geben. Damit geht jedoch nicht der Anspruch einher, sämtliche Ambivalenzen des Governance-Begriffs auflösen zu wollen. Auch beim Lenkungsbegriff bleiben verschiedene am Governance-Begriff kritisierte Uneindeutigkeiten bestehen, wie beispielsweise die unklare Subjekt/Objekt-Unterscheidung, indem die Adressaten der mittels ,Governance' beschriebenen Handlungen gleichzeitig auch selbst an deren Entwurf und Durchsetzung beteiligt sind (vgl. Mayntz 2005; Offe 2008).

Lenken steht in der hier vertretenen Auffassung für sowohl gesetzliche als auch selbstregulatorische und freiwillige Regulierungsansätze, ebenso wie für partizipative Ansätze und Abschätzungsaktivitäten. Der Governance-Begriff hat seinen Ursprung in der Ökonomie, wo er für institutionelle Regelungen in Unternehmen steht und in der Politikwissenschaft, wo er zur Analyse internationaler Beziehungen verwendet wird (vgl. Benz 2004; Schuppert 2008). Governance wird in zwei verschiedenen theoretischen Kontexten benutzt: einerseits allgemein gesellschaftstheoretisch als Oberbegriff für die verschiedenen Formen sozialer Handlungskoordination (Hierarchie, Markt, Gemeinschaft, Organisationen). Andererseits steht Governance im Kontext internationaler und nationaler Politik auch zur Bezeichnung nicht-hierarchischer und nicht ausschließlich staatlicher Formen von Regulierung (vgl. Benz, et al. 2007; Mayntz 2004). Inzwischen dominiert ein gemeinsames Verständnis von Governance als für die ,Koordination staatlicher und nichtstaatlicher Akteure in und durch 
Regelungsstrukturen' (vgl. Schuppert \& Zürn 2008, 23). ${ }^{10}$ Der Lenkungsbegriff umfasst hier also Steuerungs-Formen, die sowohl auf gesetzlicher Regulierung basieren als auch solche, die auf informellen Regulierungsansätzen beruhen. Zudem umfasst er sanfte Ansätze mit Steuerungscharakter, wie Technikfolgenabschätzungen, Risikoanalysen, Risikomanagementansätze ebenso wie partizipative und kooperative Formen von Kommunikation und Verhandlung. Auch umfasst der Lenkungsbegriff selbstregulatorische Ansätze, die sich in der Politikwissenschaft unter dem englischen Begriff der ,new governance' etabliert haben. ${ }^{11}$ Governancekonzepte decken also unterschiedlichste Phänomene moderner Gesellschaften im Kontext einer veränderten Rolle des Staates in neoliberalen Regierungsformen ab (Offe 2008; Schuppert 2008). Im Kontext von Governance wird Regulieren als eine die gesamte Gesellschaft betreffende Aufgabe aufgefasst und die beobachtete Zunahme regierungsexterner Steuerung als Verschiebung von ,Government' zu ,Governance' beschrieben (Rhodes 1997). Abhängig zur analytischen Perspektive wird dieser Wandel von gesetzlicher zu gesellschaftlicher Regulierung als ,governance turn“ (Borràs 2008; Rose \& Miller 1992), ,deliberative turn` (Hagendijk \& Irwin 2006), ,qualitative turn“ (Kearnes 2009; Kearnes, et al. 2006a; Kearnes \& Rip 2009) oder als ,turn from modernist to post-modernist forms of statecraft' (Gottweis $\&$ Petersen 2008) beschrieben. Wie dies für den Kontext der Governance beschrieben worden ist, steht auch der Lenkungsbegriff für eine Auffasung von Regulieren als eine die gesamte Gesellschaft betreffende Aufgabe (Schuppert \& Zürn 2008).

Insbesondere im Kontext der Nanotechnologie steht der hier als Lenken übersetzte Governance-Begriff für eine Fülle unterschiedlichster Steuerungs- und Regulierungsansätze, die außerhalb der traditionellen gesetzlichen Regulierung entstehen. Zudem steht ,governance of nanotechnology' für eine normativ geprägte Haltung, wie die Nanotechnologie zu verwalten, zu regulieren, zu definieren und zu steuern ist - sei es mit einer ,nach-

10 Im Kontext von Nanotechnologie spricht (Lösch 2014, 188) von , allen Aktivitäten (mit Regelungsanspruch), die unter den Governancebegriff subsumiert werden. Gerade durch seine Unschärfe eignet sich der Governancebegriff zur Beschreibung von Selbstregulierungsprozessen in emergierenden Technikfeldern wie der Nanotechnologie (ibid. 189).

11 Zum Begriff der new governance, siehe beispielsweise de Búrca and Scott (2006), Peters (2011), Peters and Pagotto (2006), Lyall and Tait (2005), Eberlein and Kerwer (2004), Borràs and Jacobson (2004), Scott and Trubek (2002). 
haltigen' (Florini, et al. 2006; Meili 2006), ,innovativen' (Curran \& al. 2006), ,verantwortungsvollen' (COM 2008a; NIA 2008) - oder einer ,antizipatorischen' (Barben, et al. 2008) Governance (vgl. Kaiser 2015).

Wichtig ist in diesem Kontext auch die Abgrenzung zwischen Governance beziehungsweise dem hier verwendeten Lenkungsbegriff und der Steuerung. Während Steuerung hauptsächlich Akteure fokussiert, also einem korporativen und akteurzentrierten und auch hierarchischen Ansatz folgt, meint Governance und der hier verwendete Lenkungsbegriff eher Strukturen und wird als strukturzentrierter, institutionalistischer und kooperativer Ansatz verstanden, der nach der Beschaffenheit von Regelungsstrukturen fragt, in denen öffentliche und private, hierarchische und netzwerkartige Formen der Regelung zusammenwirken (Mayntz 2005; Mayntz 2008; Mayntz \& Scharpf 1995). Oder anders gesagt, GovernanceAnsätze lösen ältere, eher eindimensionale und unilineare Steuerungs-, Planungs- und Entscheidungstheorien ab und ersetzen sie durch netzwerkförmige, reflexive und rekursive Ansätze (Bora 2012, 345). Der Übergang von Steuerung zu Governance erfolgt nach Bora (2012) als Wandel von einer engen Handlungsorientierung zu einer breiten Strukturperspektive. ${ }^{12}$

Im nächsten Kapitel wird die kulturanalytische Deutung des Lenkungsbegriffs erläutert. Dabei stehen vergleichende Ansätze der sozialwissenschaftlichen Wissenschafts- und Technikforschung, wie das Konzept der Wissenskulturen (Knorr Cetina 2002) und dasjenige der politischen Kultur, (Jasanoff 2005) im Zentrum.

\subsection{Lenkungskulturen}

Das dieser Arbeit zugrunde liegende analytische Raster orientiert sich einerseits an dem von Keller (2011) vorgeschlagenen Ansatz der wissenssoziologischen Diskursanalyse (siehe Kapitel 1.1) und andererseits an den vergleichenden Kulturanalysen der sozialwissenschaftlichen Wissenschafts- und Technikforschung. Die hier berücksichtigten Ansätze kombinieren das von Knorr Cetina (2002) zum Vergleich der akademischen Wissensproduktion in Spitzenforschungsfeldern wie der Hochenergiephysik

12 Dabei beschreibt der Governance Begriff Formen sozialer Handlungskoordination, speziell kooperative Formen im Gegensatz zu hierarchischer Steuerung. Auch werden Steuerung durch Netzwerke, partizipative Beteiligungsforen und die reflexive Beobachtung unter dem Begriff Governance subsumiert (ibid., 346). 
und der Molekularbiologie entwickelte Konzept der Wissenskulturen mit dem von Jasanoff (2005) für das Feld der sozialwissenschaftlichen Wissenschafts- und Technikforschung weiterentwickelte Konzept der politischen Kultur. Dieses ursprünglich in der Politikwissenschaft verbreitete Konzept hat Jasanoff auf komparative poststrukturalistische Analysen angepasst und damit den Vergleich des politischen Diskurses der Gentechnik in westlichen Industrienationen untersucht. Sowohl Knorr Cetina als auch Jasanoff stützen ihre kulturellen Vergleichsansätze auf drei analytische Kategorien ab: Diese umfassen 1) eine soziale bzw. personenbezogene, 2) eine inhaltliche bzw. wissensorientierte und 3) eine Dimension, welche auf die verwendeten Ansätze, Methoden und Praktiken fokussiert.

Knorr Cetina (2002) beschreibt eine Wissenskultur als ein bestimmtes Zusammenwirken dreier Maschinerien: einer empirischen (im Sinne von Instrumenten, Methoden und Praktiken), einer ontologischen (im Sinne von Prozessen, Objektbeziehungen und Inhalten) und einer sozialen Maschinerie (im Sinne sozialer Beziehungen oder Personen). Eine Wissenskultur entsteht also in spezifischen Konstellationen, die sich aus der Anwendung ausgewählter Instrumente, Methoden, Praktiken und aus der Wahl spezifischer Forschungsprozesse ergibt. Diese Konstellationen werden durch die jeweiligen Objektbeziehungen und die untersuchten Inhalte ebenfalls mitgeprägt. Diese Objektbeziehungen werden wiederum durch die jeweils zu bestimmten Zeitpunkten an ausgewählten Orten vorhandenen sozialen Beziehungen zwischen Forschenden, Vorgesetzten und der Fachgemeinschaft geprägt (ibid.).

Jasanoff (2005, 23 ff.) baut ihr Konzept der politischen Kultur auf den Kategorien Verhandlung, Darstellung und Partizipation aus. Unter der Kategorie Verhandlung meint sie die Ebenen des politischen Diskurses, deren Leistung und Grenzen. Als Darstellung bezeichnet sie die Mittel, durch welche bestimmte Stimmen im politischen Prozess hörbar gemacht werden und den Einfluss, den die politische Einbindung auf die Wahrnehmung bestimmter Themen ausübt. Unter der Kategorie Partizipation versteht sie die involvierten Akteure und Anspruchsgruppen. Dabei wird das politikwissenschaftliche Verständnis von politischer Kultur um eine machtanalytische Perspektive und um Fragen der Legitimität erweitert. Nach Jasanoff (2005) spielen in zeitgenössischen Wissensgesellschaften auch stillschweigende Routinen der Produktion und der Bewertung von sozialem Wissen eine wichtige Rolle für die Ausprägung einer bestimmten politischen Kultur. Solche Routinen umfassen Aspekte wie intersubjektive Wahrnehmungspraktiken bestimmter Inhalte, deren Interpretationskontext 
und deren Einordnung (ibid.). Bedeutsam sind auch soziale Abgrenzungsbemühungen hinsichtlich bestimmter Konzepte wie Akzeptanz, Hierarchie und bestimmter Vorstellungen und Institutionen, deren Ansprüche, Denkweisen, Vorstellungen, Diskurse und Handlungen. Ebenso wichtig sind deren Strategien, mittels welchen sie Legitimität einfordern und aufrechterhalten. Aus dieser Perspektive entsteht politische Kultur nicht nur aus der politischen Praxis, sondern auch aus weniger expliziten kulturellen Verbindlichkeiten, welche die Politik legitimieren (Jasanoff 2005, 22).

Ausgehend von diesen Ansätzen und unter Berücksichtigung der von der wissenssoziologischen Diskursanalyse (Keller 2011) vorgegebenen Kategorien, geht diese Arbeit davon aus, dass sich Lenkungskulturen an den drei analytischen Kategorien, Akteure, Themen und Praktiken, manifestieren. Eine Lenkungskultur zeigt sich also darin, wie unterschiedliche Akteure in der Koordination ihres kollektiven Handelns bestimmte Themen besetzen und ausgewählte Praktiken anwenden. Das spezifisch Kulturelle ergibt sich dabei aus inkrementellen, vieldeutigen aber auch zufällig und situativ auftretenden Mustern und Verbindungen zwischen Akteuren, Themen und Praktiken, die in nationalen, historischen und sozialen Kontexten und in bestimmten personellen und zeitgenössischen Traditionen und Konstellationen der kollektiven Handlungskoordination entstehen. Die Interaktionen zwischen den drei Dimensionen Akteure, Themen und Praktiken stellen hier also das analytische Raster dar, mit dem die Lenkungskulturen beschrieben werden, die sich im Umgang mit Nichtwissen beobachten lassen.

Lenkungskulturen zeigen sich in dieser Arbeit an den in den jeweiligen Ländern und auf der Unionsebene von nationalen und transnationalen historischen, politischen und sozialen Kontexten geprägten aber auch an zufällig und situativ beobachtbaren personellen und thematischen Konstellationen. Die Gesellschaften der untersuchten Länder und der Europäischen Union wenden in ihrer kollektiven Handlungskoordination im Umgang mit Nichtwissen bestimmte Praktiken an. Dabei zeigt sich das spezifisch Kulturelle in den Lenkungsdiskursen über Nichtwissen in den jeweiligen Zwischenräumen und Interaktionen, die sich zwischen den involvierten Akteuren, den verhandelten Themen und den angewendeten Praktiken zeigen. Das Kulturelle liegt dabei also in den impliziten und expliziten Verbindungen zwischen den drei Kategorien Akteure, Themen und Praktiken, ihren historischen, sozialen und politischen Kontexten und in der Art, wie diese Verbindungen in den jeweiligen Kontexten zustande kommen, gelebt werden und ihre Wirkung entfalten. 
Das nachfolgende Kapitel beleuchtet den für die vorliegende Arbeit relevanten Fall der Nanotechnologie, der die empirische Grundlage dieser Studie darstellt. Dabei wird in die wissenschaftspolitische Entstehung der Nanotechnologie eingeführt und ihre Auffassung als technowissenschaftliches Phänomen erläutert.

\subsection{Die Nanotechnologie als technowissenschaftliches Phänomen}

Der größte gemeinsame Nenner der vielfältigen begrifflichen Definitionen der Nanotechnologie bezieht sich auf ihre Größe. In dieser Auffassung steht Nanotechnologie für die Manipulation von Materie auf atomarer und molekularer Ebene im Größenbereich von 1-100 Nanometern (also 10-9 Metern). ${ }^{13}$ Um ihren vielfältigen Anwendungen Rechnung zu tragen, wird verschiedentlich von den Nanotechnologien im Plural, beziehungsweise von den Nanowissenschaften und Nanotechnologien gesprochen (z.B. RS\&RAE 2004). Der der Nanotechnologie zugrunde liegende Forschungsbereich hat sich in den 1990er Jahren als interdisziplinärer Ansatz aus der Physik, der Materialwissenschaft, der Chemie und der Biologie entwickelt. Diese Disziplinen haben sich die spezifischen Eigenschaften von Materie in dieser Größenordnung für ihre Forschung und Entwicklung zunutze gemacht. Nanotechnologie steht dabei für visionäre Ansätze wie Nanoroboter und Quantencomputer ebenso wie für molekulare Systeme, auf spezifische Eigenschaften hin hergestellte kleinstskalige Materialien, miniaturisierte Systeme und gezielte Manipulationen an biologischen Systemen. ${ }^{14}$

13 Siehe dazu beispielsweise in der Ausformulierung der US-amerikanischen Nationalen Nanotechnologie Initiative (NNI) (NSTC 2000), in verschiedenen Definitionen wie (COM 2011; Decker 2006; SCENIHR 2007b; Schmid, et al. 2003) oder in Abschätzungsstudien wie beispielsweise (Paschen, et al. 2004; RS\&RAE 2004). Für einen populärwissenschaftlichen Überblick über die Nanotechnologie siehe z.B. (Boeing 2004). Lösch $(2012,173)$ bezeichnet die zur internationalen Konvention erhobene Allgemeindefinition, dass die Nanotechnologie Produkte und Entwicklungen umfasst, die durch die Manipulation von Materie in mindestens einer Dimension im Größenbereich von $<100 \mathrm{~nm}$ erzeugt werden, als , den einzigen gemeinsamen epistemischen Nenner der unter der Klammer ,Nano' zusammengefassten heterogenen Elemente' (vgl. auch Lösch 2009; Lösch 2010; Nordmann 2007).

$14 \mathrm{Zu}$ verschiedenen Visionen und Anwendungen siehe auch Kapitel 2. 
Diskursanalytisch wird die Nanotechnologie oft in den Kontext zu vorangegangenen Technikdiskursen wie diejenigen um die Gentechnik (hier insbesondere landwirtschaftliche Anwendungen bzw. die sogenannt, grüne Gentechnik') und um die Nukleartechnologie gestellt (z.B. Einsiedel \& Goldenberg 2004; Grove-White, et al. 2004; Kearnes, et al. 2006a; Mayer 2002; Mehta 2004; Wolfson 2003). Die Nanotechnologie und die grüne Gentechnik gelten zwar als äußerst unterschiedliche Technologien, die aus verschiedenen wissenschaftlichen Disziplinen mit unterschiedlichen Zielen entwickelt worden sind' (Kearnes et al. 2006a). Dennoch wird die Haltung vertreten, dass es sich bei der Nanotechnologie um eine weitere ,potenziell transformative Technologie' handelt, die sich wie die grüne Gentechnik in den späten 1970er bzw. frühen 1980er Jahren im Entwicklungsstadium befindet (ibid.). Als vergleichbar werden auch die mit der Nanotechnologie verbundenen utopischen Verheißungen, Erwartungen und distopischen Szenarien bezeichnet (Nordmann, et al. 2004). Bei den Vergleichen der beiden Technologien steht jedoch hauptsächlich die Frage im Zentrum, wie die Erfahrungen mit der Gentechnikkontroverse politische und regulatorische Debatten um die Nanotechnologie beeinflussen (Kearnes, et al. 2006a). Als eine dieser Erfahrungen aus der Gentechnikkontroverse wird der zentrale Einfluss der öffentlichen Wahrnehmung auf den kommerziellen Erfolg einer neuen Technologie gesehen. Dabei wird die Gentechnikkontroverse als ,Modell' bezeichnet, das in der Entwicklung und in der politischen Behandlung der Nanotechnologie vermieden werden sollte (Kearnes, et al. 2006a).

Mehrheitlich werden vorangegangene Technikdiskurse also als Referenzfälle dargestellt, aus welchen sich der Imperativ ergibt, Lehren für die wissenschafts- und gesellschaftspolitische Behandlung der Nanotechnologie zu ziehen (ibid.). Solche werden insbesondere im Umgang mit der Öffentlichkeit gesehen. Statt eines Aufklärungsdiskurses wird für eine frühzeitige soziale Einbettung der Nanotechnologie im Sinne einer ,konstruktiven Technikfolgenabschätzung' (Rip, Misa, Schot 1995) plädiert. Ebenfalls problematisiert wird die Haltung von Entscheidungsträgern im Gentechnikdiskurs, Ansätze und Strategien von vorangegangenen Technologien relativ unhinterfragt anzuwenden ebenso wie diejenige der Wissenschaft, unausgesprochene soziotechnische Visionen und Vorstellungen der sozialen Rolle der Nanotechnologie zu übernehmen (z.B. Kearnes, et al. 2006a). Felt (2015a) spricht dabei von ,soziotechnischen Imaginationen', die in dieser Einbettung der Nanotechnologie in den jeweiligen Ländern zu spezifischen technopolitischen Identitäten führen. 
Die Nanotechnologie wird auch als ein Phänomen beschrieben, das nicht wie traditionelle Technologien den Erfindungen und Entdeckungen in wissenschaftlichen Laboratorien entsprungen und über jahrelange Entwicklungsarbeit, Marktanalysen und gesellschaftliche Interaktionen zur praktischen Reife gelangt ist (Merz 2015; Merz \& Biniok 2014; Nordmann, et al. 2004; Schummer 2004). Vielmehr wird sie als im Kontext des internationalen Wettbewerbs um Innovationsvorherrschaft als ,vielschichtiges', ,multidimensionales', ,variables' und ,gesellschaftlich-kommunikatives Phänomen' beschrieben, das diskursiv (Lösch 2014) und in Hinblick auf zukünftige Anwendungen (Selin 2007a) konstruiert wird. Ebenfalls wird die Nanotechnologie als gezielt etabliertes politisches Projekt einer technowissenschaftlichen Schwerpunktsetzung beschrieben (Merz 2010; Schaper-Rinkel 2010b; Schummer 2009; Schummer \& Baird 2006). Als inhärent politisch werden auch die mit der zukünftigen Entwicklung der Nanotechnologie verknüpften Nanomaterialien beschrieben (Laurent 2013). Die Nanotechnologie wird auch als Sammelbegriff für ökonomisch vielversprechende Forschungsgebiete aus den Natur- und Ingenieurswissenschaften (Lösch 2012, 173; Schummer 2009) und als Schirmbegriff ,umbrella term' (Rip \& Voss 2013) bezeichnet, der einer Reihe an populärwissenschaftlichen Projektionen ,folk theories'. Als ,folk theories' beschreibt Rip die von Nanowissenschaftlern im politischen Diskurs geäußerte populärwissenschaftlichen Vorstellungen, wie ihre Angst vor öffentlichem Widerstand gegen die Nanotechnologie, die Rip (2006) als ,nanophobia-phobia' bezeichnet, oder die Vorstellung, dass sich die anfängliche Begeisterung für die Nanotechnologie vergleichbar zu derjenigen der Gentechnikkontroverse rasch in Ablehnung, wow-yuck' (Kulinowski 2004) umwandeln wird. ${ }^{15}$ Vorschub leistet. Ebenso wird die Nanotechnologie als ,nahtloses Gewebe aus Sachtechniken, Netzwerken und zukunftsbezogenen Diskursen' (Lösch 2014; Selin 2007a), bzw. als ,leerer Signifikant' beschrieben, der seine Bedeutung infolge einer Überdeterminierung durch widersprüchliche Deutungen fast vollständig verliert (Åm 2011; Wullweber 2009) und in Selbstauflösung begriffen ist (Kaiser 2015).

Die Nanotechnologie steht auch für eine Flut an lokal diversen wissenschaftlichen, politischen, wissenschaftspolitischen und kommerziellen Diskursen, welche das Forschungs-, Entwicklungs- und Innovationspoten-

15 Ebenso als ,folk theory' wird die Haltung beschrieben, dass die Nanotechnologie angesichts ihres umfangreichen gesellschaftlichen Nutzens mit übertriebenen Versprechen beworben werden soll (Rip 2006). 
zial ebenso wie potenzielle nachteilige Implikationen verhandeln. ${ }^{16}$ Diese Diskurse stehen im Zentrum der vorliegenden Analyse. Dabei dient die Nanotechnologie hier als Fall, an welchem der gesellschaftliche Umgang mit Nichtwissen dargestellt wird; beziehungsweise an dem unterschiedliche Kulturen im Lenken von Nichtwissen herausgearbeitet werden.

Die Nanotechnologie ist insbesondere in ihren Anfängen als Ansatz propagiert worden, mittels welchem die ,Welt' ,Atom für Atom', neu aufgebaut' werden soll (NSTC 1999). ${ }^{17}$ Durch die damit implizierte untrennbare Verknüpfung der wissenschaftlichen Beschreibung der Reaktionsweise von Materie im Nanoskalenbereich mit dem technischen Eingriff in die Gestaltung dieser Materie, gilt die Nanotechnologie als Paradebeispiel einer Technowissenschaft (Nordmann 2005).

Der Begriff der Technowissenschaft wird dabei unterschiedlich verwendet. Im Kontext der Laborstudien der sozialwissenschaftlichen Wissenschafts- und Technikforschung ist er von Bruno Latour (Latour 1987) geprägt worden. Latour (1987) beschreibt damit die durch technische Geräte und Artefakte unterstützte spezifische Art der Wissensproduktion in naturwissenschaftlichen Laboratorien. In Weiterentwicklung dieses Ansatzes vertreten Law und Mol (2001) die Ansicht, dass sämtliche technischen Geräte und Elemente, die wissenschaftliche Inhalte prägen, technowissenschaftliche Artefakte darstellen. Demnach finden Technowissenschaften nicht nur in naturwissenschaftlichen Laboratorien, sondern darüberhinaus in den verschiedensten fluiden Formen globaler Netzwerke statt (Law \& Mol 2001).

In der Folge wird der Begriff von unterschiedlichen Strömungen der sozialwissenschaftlichen Wissenschafts- und Technikforschung aufgegriffen und weiterentwickelt. Insbesondere etabliert sich die Auffassung einer Hybridisierung und Untrennbarkeit der früher klar voneinander abgrenzbaren Wissenschaft, die natürliche Phänomene beobachtet und die Natur beschreibt, und der Technologie, die in diese eingreift (Nordmann 2005). Die Auffassung von Technowissenschaft als Verschmelzung und Verwischung von Grenzen wird in der Folge bestimmend in der Darstellung technowissenschaftlicher Phänomene und entspricht auch der dieser Studie zugrunde liegenden Verwendung. Haraway (1991) beschreibt beispielsweise am Fall der Krebsmaus eine ,technowissenschaftliche Kultur', die in einer transna-

16 National und regional unterschiedliche lokalen Konfigurationen neuer Technikfelder, zeigen sich auch am Fall der Nanotechnologie (vgl. Merz \& Sormani 2014).

17 Vergleiche dazu auch Nordmann (2003) und Jones (2004). 
tional kapitalistischen Weltordnung der späten Moderne, Unterscheidungen wie solche zwischen Technologie und Wissenschaft aber auch zwischen Mensch und Nichtmensch, zwischen Natur und Gesellschaft, zwischen Tatsachen und Artefakten als auch zwischen Subjekten und Objekten verwischt und hybride Figuren (cyborgs) hervorbringt (vgl. auch Michael 2006, 31).

Als Weiterentwicklung dieser Hybridisierung hat Michael (2006) den Begriff des technowissenschaftlichen Bürgers (technoscientific citizen) eingeführt, der nicht nur die Durchdringung technowissenschaftlicher Ansätze im Alltagsleben durch Alltagstechnologien und Artefakte beschreibt, sondern auch für die Verwischung der Grenze zwischen Technik, Wissenschaft und Gesellschaft steht (vgl. dazu auch Felt, et al. 2007; Irwin \& Michael 2003; Nowotny, et al. 2001). Als technowissenschaftliches Wissen beschreibt schließlich Nordmann (2010) eine neue Wissensart; das ,fertigkeitsorientierte' Wissen. Diese durchdringt das in den grundlagenforschenden Wissenschaften traditionell hergestellte epistemische Theoriewissen. Als fertigkeitsorientiertes Wissen wird also die internationale Vernetzung von individuellen Fertigkeiten aufgefasst, die sich auch in der Verschmelzung von traditionell disziplinär getrennt produziertem Wissen manifestiert. In diesem Kontext spricht Schaper-Rinkel (2006, 479) von einer ,Amalgamisierung von Wissenschaft, Gesellschaft, Politik und Ökonomie'. Technowissenschaften lassen sich also nicht durch neuartige Theorien, Methoden oder Interventionen charakterisieren, sondern definieren sich durch ein Konglomerat von zusammenwirkenden Techniken, Praktiken, Diskursen und Visionen, die eine ,technowissenschaftliche Kultur' (Nordmann 2011) ausmachen (Lösch 2014, 13).

Am Beispiel der Nanotechnologie, deren Forschungsgegenstand weder der Natur noch der Technik oder der Gesellschaft bzw. der Kultur zuordenbar ist, lässt sich der mit dem Begriff der Technowissenschaft beschriebene grundsätzliche Wandel der Wissenschaftskultur von einer beobachtenden, beschreibenden Haltung hin zu hybriden Formen und Entstehungsbedingungen des gezielten Eingriffs besonders gut zeigen (Felt 2010; Lösch 2014). Hinsichtlich ihrer Herkunft, ihrer Zukunft als auch der Bandbreite ihrer Anwendungen stellt die Nanotechnologie also eine höchst unbestimmte Technologie dar. Aus diesem Grund sieht diese Studie das gesellschaftspolitische Grundproblem in der Frage nach dem Umgang mit dem der Nanotechnologie inhärenten Nichtwissen. So setzt sich die vage und uneindeutige begriffliche Verortung der Nanotechnologie im von hohen Ungewissheiten und Unsicherheiten geprägten politischen Diskurs 
zu potenziellen Risiken und ihrer Regulierung fort (Lösch 2012, 173). Die Regulierung der Nanotechnologie wird als eine neue Art der losen und flexiblen Regulierung bezeichnet, die von freiwilligen Lenkungsmaßnahmen und ,intermediären' Institutionen wie Anspruchsgruppengremien geprägt ist. Deren Ziel wird als darin liegend beschreiben, Konflikte vorwegzunehmen und zu entleeren, bevor sie entstehen (Åm 2011; Kaiser 2015; Wullweber 2009).

Gegenstand des nachfolgenden Kapitels sind die der Untersuchung des gesellschaftlichen Umgangs mit Nichtwissen am Fall der Nanotechnologie zugrunde liegenden Thesen und Fragestellungen.

\subsection{Thesen und Fragestellung}

Die Beobachtung einer verstärkten Fokussierung auf Nichtwissensfragen in ökologischen und technowissenschaftlichen Diskursen wirft zwei Fragekomplexe auf: Der erste fragt nach der Ursache oder der Begründung dieses Fokuswandels. Der zweite stellt die Frage nach der Konsequenz oder dem gesellschaftlichen Umgang mit dem neu im Fokus von umweltund technowissenschaftlichen Diskursen stehenden Nichtwissen. Der verstärkte Fokus auf Nichtwissen hat vielfältige soziologische Erklärungsansätze produziert, wie beispielsweise sein Auftreten in umweltrelevanten, auch als ,ökologische Kommunikation' (Luhmann 1986) bezeichneten Kontexten. Damit einhergehend wird Nichtwissen mit dem Auftreten von unvorhergesehenen Störfällen verknüpft, mit großtechnischen Katastrophen und mit der an Komplexität gewinnenden technowissenschaftlichen Entwicklung (Beck 1986). Ebenso als Nichtwissensthemen aufgefasst werden die damit verknüpften intensivierten Selbstreflexionen im Zuge der Entwicklung einer reflexiven Moderne (Beck, et al. 1996), die veränderten Machtbeziehungen im politischen Diskurs (Rose \& Miller 1992) und die Verlagerung von transdisziplinärer Wissensproduktion in die Gesamtgesellschaft (Gibbons, et al. 1994; Nowotny, et al. 2001). Demgegenüber liegt der Fokus dieser Studie auf einem derzeit noch weniger intensiv beforschten Fragekomplex; dem gesellschaftlichen Umgang mit Nichtwissen.

Gegenstand dieser Studie ist also nicht das Nichtwissen an sich, seine Wirkungen, Funktionen und Entstehungsbedingungen. Vielmehr wird hier am Beispiel der Nanotechnologie gezeigt, wie Gesellschaften, ihre Institutionen, Organisationen und Akteure Nichtwissen verhandeln und damit 
einen Umgang suchen. Dies geschieht vor dem Hintergrund der mit der Nanotechnologie einhergehenden Konstellationen von Ungewissheit, Unsicherheit, Unklarheit und Nichtvorhersehbarkeit potenzieller nutzbringender Anwendungen und nachteiliger Implikationen. Die Verschiebung des gesellschaftlichen Fokus in technowissenschaftlichen Diskursen von Wissen zu Nichtwissen und die damit einhergehenden neu auftretenden Verantwortlichkeiten und Konstellationen von Anspruchsgruppen, Arten der Expertise und Kooperationen haben zu einer Reihe von gesellschaftspolitischen Veränderungen geführt. Diese äußern sich — so eine zweite These — in spezifischen, neuartigen Verhaltensmustern bzw. Strategien im Umgang mit Nichtwissen. Solche umfassen einen signifikanten Anstieg von Abschätzungsbemühungen, von selbstregulatorischen und nicht bindenden Regulierungsansätzen und von Ansätzen der partizipatorischen Einbindung von Anspruchsgruppen und der Zivilgesellschaft in die wissenschafts- und technikpolitische Entscheidungsfindung. Diese Strategien haben das gemeinsame Ziel, die Nanotechnologie ,regierbar zu machen' (Kaiser 2015), oder wie in dieser Studie argumentiert wird, Nichtwissen zu lenken.

Daraus ergibt sich in Nichtwissensdiskursen also ein gesellschaftliches Bedürfnis nach Lenkung, bzw. ein Lenkungsdispositiv, das darauf abzielt, Nichtwissen definierbar, kalkulierbar und regulierbar zu machen. Dieses Lenkungsdispositiv umfasst die drei zentralen Strategien; 1) das Abschätzen, 2) das Regulieren und 3) die Partizipation. Diese Strategien, die analytisch als Diskurse behandelt werden, sind in inkrementellen, vieldeutigen aber auch zufällig und situativ auftretenden Mustern und Verbindungen zwischen den involvierten Akteuren, den von ihnen diskutierten Themen und den angewendeten Praktiken in Nichtwissensdiskursen verwoben. Diese Verwebungen zwischen Akteuren, Themen und Praktiken in und zwischen den jeweiligen Strategien des Umgangs mit Nichtwissen, formen eine jeweils in den untersuchten Kulturräumen wahrnehmbare spezifische Lenkungskultur des Umgangs mit Nichtwissen.

Die diskursanalytische Untersuchung der Frage nach den Lenkungskulturen des Nichtwissens umfasst verschiedene Fragekomplexe. So wird grundsätzlich nach den damit verbundenen Diskursen gefragt, ihrem Entstehen, ihrem Verlauf, möglicherweise auch nach ihrem Verschwinden und den aus den Diskursen entstandenen Dispositiven. Innerhalb der untersuchten Diskurse fragt die vorliegende Untersuchung nach den involvierten Akteuren, den von ihnen verhandelten Themen und den verwendeten Praktiken. Im Zentrum der Analyse steht also die Frage, wer in Nichtwis- 
sens- und Lenkungsdiskursen was mit wem und wie verhandelt und welche sozialen Phänomene damit erklärt werden können. Konkret stellen sich die folgenden vier Fragekomplexe:

1) Fragen nach den involvierten Akteuren: Welche Akteure besetzen mit welchen Ressourcen, Interessen, Strategien die Sprecherpositionen? Wer ist Träger, Adressat und Publikum des Diskurses? Wer interagiert mit wem? In welchen sozialen Konstellationen werden Nichtwissen und Lenkungsfragen verhandelt? Welche Rolle nehmen die spezifischen Akteure in technowissenschaftlichen Diskursen ein und wie werden diese durch die diskurseigenen Konstellationen verändert?

2) Fragen nach den Themen und Inhalten: Welche Inhalte werden vermittelt? Welches Wissen (Deutungen und Problemlösungen) wird erzeugt und verbreitet? Welche Phänomenbereiche werden dadurch wie konstituiert? Wie werden Nichtwissen und Lenkungsfragen thematisiert? Wie werden ursprünglich genuin wissenschaftliche, politische, wirtschaftliche und zivilgesellschaftliche Fragen und Aspekte von den verschiedenen Akteuren in technowissenschaftlichen Diskursen zusammengebracht und wie fließen diese zurück in die relevanten gesellschaftlichen Bereiche wie Wissenschaft, Politik, Wirtschaft und Nichtregierungsorganisationen?

3) Fragen nach Ansätzen, Methoden und Praktiken: Wie, wo, mit welchen Praktiken und Ressourcen wird ein Diskurs produziert? Welche kommunikativen Mittel und Strategien werden eingesetzt? Wie werden Nichtwissen und Lenkungsfragen in bestimmte Praktiken umgesetzt? Wie beeinflussen die technowissenschaftlichen Diskurse die von bestimmten Akteuren verwendeten Praktiken und wie beeinflussen die Praktiken spezifische thematische Konstellationen? Welche Unterschiede und Veränderungen in den Verbindungen und Konstellationen zwischen Akteuren, verhandelten Themen und den angewendeten Praktiken lassen sich beobachten?

4) Übergreifende Fragen nach dem Diskurs, seinem Verlauf, den Bezügen, den daraus generierten Dispositiven, der sozialen Wirkung und den länderspezifischen Unterschieden: Was sind die entscheidenden Ereignisse im Verlauf der untersuchten Diskurse, wie verändern sie sich mit der Zeit? Wie unterscheiden sie sich in den untersuchten Nationen bzw. Kulturräumen? In welchem Bezug stehen die untersuchten Diskurse $\mathrm{zu}$ anderen, historisch vorangegangenen Technikdiskursen und zum sozialen Kontext? Welche Aushandlungsprozesse finden in 
den Diskursen statt? Wie schlagen sich Diskurse in Dispositiven nieder? Wie formen die untersuchten Diskurse ihren Gegenstand, ihre Begriffe und die Strategien? Welche gesellschaftlichen Folgen und Machwirkungen gehen von den untersuchten Diskursen aus?

Aus diesen vier Fragekomplexen ergibt sich also die nachfolgende diskursanalytische Untersuchung nach den Lenkungskulturen des Nichtwissens. Diese Analyse umfasst die inkrementellen, vieldeutigen aber auch zufällig und situativ auftretenden Muster und Verbindungen zwischen dem Verlauf, den Bezügen und den Dispositiven, die sich aus einem Diskurs ergeben und den involvierten Akteuren, den diskutierten Themen und den angewendeten Praktiken. Die Lenkungskulturen des Nichtwissens werden in einem internationalen Vergleich zwischen den Vereinigten Staaten, der Europäischen Union, Großbritannien und Deutschland untersucht.

\subsection{Gliederung des Bandes}

Die Gliederung des Bandes verläuft entlang der drei für den gesellschaftlichen Umgang mit Nichtwissen im Nanotechnologiediskurs identifizierten Strategien, die ihrerseits jeweils eigene Diskurse generieren: dem Abschätzungs-, dem Regulierungs- und dem Partizipationsdiskurs. Diese drei Hauptkapitel gliedern sich wiederum entlang des internationalen Vergleichs. Jedes der drei Hauptkapitel wird mit einem Exkurs zu einer paradigmatischen Maßnahme eingeleitet. Am Beispiel dieser Maßnahme werden die zentralen Thesen des jeweiligen Kapitels bereits vorweg erläutert. Anschließend wird dieser jeweils länderspezifisch entlang der oben genannten analytischen Kategorien, 1) der Themen und damit verknüpft der Frage: Was wird abgeschätzt/reguliert/in partizipativen Anlässen diskutiert?, 2) der Akteure und der damit verbundenen Frage: Wer ist in den Abschätzungs-/Regulierungs-/Partizipationsdiskurs involvert?) und 3) der Praktiken und der damit einhergehenden Frage: Wer wendet welche Praktiken an? analysiert. Ebenso Gegenstand der Untersuchung sind die zufällig und situativ beobachtbaren personellen und thematischen Konstellationen, deren inkrementelle Verbindungen und Interaktionen, die sich im Abschätzen und dem Regulieren des mit der Nanotechnologie verbundenen Nichtwissens und in der Beteiligung von Anspruchsgruppen und der Zivilgesellschaft beobachten lassen. 
Im Kapitel 2 folgt die Analyse des Abschätzungsdiskurses. Dabei wird gezeigt, dass sich in der Abschätzung der Nanotechnologie drei zentrale Verschiebungen bzw. thematische Erweiterungen zeigen:

1) Von Innovationen zu Implikationen

2) Von einer breiten und vieldeutigen Nanotechnologie zu klar eingrenzbaren Nanomaterialien

3) Von der Analyse übergreifender ethischer, rechtlicher und sozialer Implikationen (ELSI) zu Umwelt-, Gesundheits- und Sicherheitsaspekten (EHS)

Wie in diesem Kapitel weiter gezeigt wird, führen diese Verschiebungen zu einem Fokuswandel im Abschätzungsdiskurs über Nichtwissen. So muss nicht mehr länger über Unbekanntes/Ungewisses oder Unklares verhandelt werden, sondern die Abschätzung, realer' Probleme und bekannter Konzepte, wie dasjenige der ,Toxizität' und diejenige bestimmter Anwendungen wie bei Lebensmitteln, Arzneimitteln und Kosmetika wird möglich. Gleichzeitig lässt sich im Kontext von Nichtwissen der Verlust eindeutig zuordenbarer Zuständigkeiten bzw. Verantwortlichkeiten zu bestimmten gesellschaftliche Institutionen feststellen. So lässt sich eine gesellschaftliche Distribution der Abschätzung feststellen, welche zu einer signifikanten Ausweitung der traditionell auf Institutionen aus Politik, Wissenschaft und Wirtschaft eingegrenzten Abschätzungsexpertise geführt hat.

Kapitel 3 behandelt den Regulierungsdiskurs. Hier stehen Beispiele wie die umstrittene Frage bezüglich der Anwendbarkeit der bestehenden Umwelt- und Chemikalienregulierung auf Nanomaterialien sowie die an Bedeutung gewinnende Regulierung durch nicht-nationalstaatliche Akteure, wie internationale Organisationen (z.B. die OECD) im Zentrum der Analyse. Ebenso bedeutsam sind durch regierungsexterne Akteure ergriffene selbstregulatorische Ansätze.

An diesen Beispielen wird gezeigt, dass eine Korrelation zwischen dem $\mathrm{Ma} ß$ an Nichtwissen und dem Formalisierungsgrad der Regulierung besteht. Gesetzliche Maßnahmen werden also nur unter Ausklammerung des Nichtwissens anwendungs- und produktorientiert getroffen, wie beispielsweise im Fall der Regulierung von Nanosilber unter der Pestizidgesetzgebung durch die US-amerikanische Umweltbehörde oder in demjenigen der Regulierung von Nanomaterialien in Kosmetika und Lebensmitteln auf Ebene der europäischen Union. Die freiwilligen und selbstregulatorischen Maßnahmen, die in Bereichen ergriffen werden, in denen ein hohes Maß 
an Nichtwissen besteht, zeichnen sich dabei durch eine doppelte Unverbindlichkeit aus; so wird in solchen Maßnahmen häufig weder der zu regulierende Gegenstand klar umrissen, noch ihre Umsetzung gewährleistet.

Kapitel 4 analysiert den Partizipationsdiskurs. Hier wird das Fazit gezogen, dass Partizipation in Nichtwissensdiskursen gerade am Nichtwissen scheitert. Dies betrifft sowohl die Partizipation von Anspruchsgruppen als auch derjenige der Zivilgesellschaft. Diese Studie zeigt, dass die Anspruchsgruppenpartizipation als ausgeweitete Form des traditionellerweise in der Politik verankerten Expertengremiums an Bedeutung gewinnt. Gleichzeitig hat sich jedoch auch gezeigt, dass den meisten Anspruchsgruppengremien die Konsensfindung schwerfällt und dass sie insbesondere in Nichtwissensfragen kaum über allgemeine und abstrakte Ergebnisse hinausgehende Erkenntnisse generieren, deren Übersetzung in die Politik nahezu unmöglich ist. Damit ist selbst die Wirkung von Gremien begrenzt, die politisch institutionalisiert sind.

Die zivilgesellschaftliche Partizipation hat insbesondere im britischen Nanotechnologiediskurs einen intensiven Boom erlebt. Doch diese unterliegt in Nichtwissensfragen im Kontext einer sich neu etablierenden Technologie dem Dilemma, dass durch das hohe Maß an Unbestimmtheit der wissenschaftlichen, technologischen und technikpolitischen Fragen das öffentliche Interesse an Partizipation begrenzt ist. Damit lässt sich die Zivilgesellschaft in Phasen der Entwicklungsoffenheit neuer Technologien kaum in deren Lenkung einbinden, obwohl hier die Gestaltbarkeit der Technologie am höchsten wäre. Damit gilt auch bei der Partizipation die für den Abschätzungs- und den Regulierungsdiskurs formulierte These, dass der jeweilige Lenkungsansatz nur dann funktioniert, wenn das Nichtwissen ausgeklammert wird. 